\title{
Optimal Sliding-Window Strategies in Networks with Long Round-Trip Delays
}

\author{
Lavy Libman and Ariel Orda \\ Dept. of Electrical Engineering \\ Technion - Israel Institute of Technology, Haifa, Israel 32000 \\ \{libman@tx, ariel@ee\}.technion.ac.il
}

\begin{abstract}
A method commonly used for packet flow control over connections with long round-trip delays is "sliding windows". In general, for a given loss rate, a larger window size achieves a higher average throughput, but also a higher rate of spurious packet transmissions, rejected by the receiver merely for arriving out-of-order. This paper analyzes the problem of optimal flow control quantitatively, for a connection that has a cost per unit time and a cost for every transmitted packet (these costs can have generic interpretations, not necessarily in terms of money). The optimal strategy is defined as one that minimizes the expected cost/throughput ratio, and is allowed to transmit several copies of a packet within a window. We derive bounds on the performance of the optimal strategy; in particular, we show that the optimal cost/throughput ratio increases merely logarithmically with the time price. We present a method for computing the optimal strategy, and demonstrate that a simple and efficient 'greedy' algorithm is sufficient to find a near-optimal solution.
\end{abstract}

\section{INTRODUCTION}

A common method for packet flow control over network connections, used both in the data-link and the transport layers, is sliding windows [1]. In this method, the receiver regularly reports to the sender the index of the next-expected packet, thereby acknowledging all the packets up to that index. The sender may transmit up to a certain number of packets, called the window size, beyond the last acknowledged packet; if a packet is not acknowledged within a certain 'timeout' period (ideally aimed to be the connection round-trip time, or slightly higher), the window is retransmitted from that packet on. In its pure form, this scheme implies that packets must arrive to the destination in order. While the receiver may temporarily keep out-of-order packets in a buffer, this does not affect the connection's performance unless the protocol is extended to allow selective, rather than cumulative, acknowledgments [2], [3]. Such extensions are not universally implemented, and even when they are, the space allocated to hold such out-of-order packets is, typically, not very large. Therefore, on a coarser level, the packet stream still has to arrive in order, allowing exceptions only to a limited extent.

Since a lost packet may trigger a retransmission of up to an entire window, its negative effect on throughput is not only due to the loss itself, but due to the time wasted in waiting for the acknowledgment as well. This effect is more severe when the connection's round-trip time (more precisely, the timeout)

\footnotetext{
*This research was supported by the Israeli Ministry of Science.
}

is long compared to the transmission time of a packet; such a connection is said to have a large bandwidth-delay product. A good example is a geostationary satellite link, with a roundtrip propagation delay of roughly 0.25 seconds, used within a high-speed connection where a packet transmission typically takes a fraction of a millisecond; the delay-bandwidth product is then measured in thousands.

Assuming that packet losses are independent (e.g. caused by white noise or a randomized discarding policy along the connection path, such as RED [4]), and that transmission of a window takes less than the round-trip delay, the throughput can be improved considerably by retransmitting some or all of the packets several times within the window itself (rather than just after a timeout, as in 'classic' sliding window schemes), as this increases their initial probability of successful arrival. For the rest of the paper, we extend the definition of the window size to include all such transmissions, counting each one separately whether it is a new packet or a copy of a previous one. We define a sliding-window strategy to be a rule that specifies how many copies of each packet, relative to the start of the window, are transmitted and in what order; in particular, it also specifies the window size. We mention at this point that alternative methods, such as forward error correction (FEC), can be used within this framework instead of simple retransmissions; we comment more on this later.

In general, for a given packet loss rate, transmitting more packets in a window - whether new ones or more copies of the same - increases the expected number of successful packets in every round-trip period, and, hence, the long-term throughput (at any rate, so long as the total window transmission time remains below the round-trip time). However, a larger window also increases the average rate of duplicate and out-of-order packets, which needlessly contributes to the network load. Thus, selection of a window size constitutes a tradeoff between these conflicting goals. To quantify this tradeoff, we associate with the connection a 'cost' per unit time and a 'cost' per packet transmission, and define the optimal strategy as one that minimizes the average cost/throughput ratio over time. We point out that these costs can have various interpretations, and should not be taken literally as money charges [5]. For example, the time cost may be associated with the disutility incurred by the application due to increased delay, and the transmission cost may be related to the energy consumption of a mobile device. Similarly, a 'social' (e.g. TCP-friendly) 
sender that refrains from retransmitting to avoid loading the network for others behaves as if it had a high per-transmission cost.

In 'classic' sliding windows, the sender transmits each packet in the window once, and the optimal strategy computation thus reduces to a trivial optimization of a single parameter (the window size). When each packet may be (re-)transmitted several times within a window, the problem becomes much more interesting. Finding the optimal strategy can then be viewed as being composed of two subproblems: an 'outer' problem of finding the optimal window size $N$, depending on the time and packet transmission costs; and an 'inner' problem of optimally distributing a total 'budget' of $N$ transmissions among the packets in a window, which, for a given $N$, no longer depends on the costs. A salient feature of the resulting solution is that not all packets are transmitted an equal number of times: earlier packets in every window get more copies transmitted than later ones, in accordance with their 'importance' (e.g., the loss of the first packet in a window results in the loss of the entire window even if later packets arrive correctly, while the reverse is not true).

In this paper, we present a detailed analysis of optimal sliding-window strategies, following the above decomposition to the 'outer' and 'inner' subproblems. It turns out that the inner problem, of deciding which packet copies to transmit for a given window size $N$, involves nontrivial combinatorial optimization, and we explore in detail its properties, derive bounds on the solution's performance, and suggest efficient approximation methods. In particular, we demonstrate that a simple 'greedy' algorithm attains nearly-optimal solutions, and proceed to extend it for the outer problem (of finding the optimal window size) as well, thus establishing an integrated solution algorithm for the strategy optimization problem. Finally, we show that the cost/throughput ratio increases only logarithmically in the time price; this is a significant improvement of the linear dependence achievable by 'classic' sliding windows.

Our current study analyzes optimal strategies limited to simple retransmissions only. A potentially better scheme for increasing the success probability of a group of packets is that of forward error correction (FEC) coding; generally, a $(n, k)$ FEC code encodes a group of $k$ packets into $n>k$ 'copies', so that any $k$ successful ones allow reconstructing the original data. We wish to emphasize that the ideas presented in this paper are not inconsistent with FEC coding, but rather complement it. If the code parameters are fixed (e.g. in a lower layer), our analysis can be readily applied by treating each encoded block as a "super-packet" with the appropriate loss probability. If the code can be controlled, the problem becomes that of finding an optimal coding strategy, which, though more complex, is based essentially on the methodology introduced here, except that the number of retransmissions is replaced by the notion of coding redundancy. In particular, it is to be expected that the optimal strategy would use higherredundancy coding for the first packets in every window than for later ones.
The special concerns raised by connections with large delaybandwidth products in general, and satellite links in particular, have attracted considerable research in recent years, e.g., [6], [7], [8], [9], [10]. Most of these studies are in the context of the widely-used TCP protocol and study how to improve its performance, either by tuning existing features [7], [8], or by introducing extensions, such as explicit congestion notifications [10]. Considerable attention has also been devoted to FEC coding that is able to adapt to higher-layer protocol requirements, and to TCP in particular (e.g. [11], [12], [13]). None of these works, however, suggested improvements of the sliding-window mechanism itself. In fact, to the best of our knowledge, the idea of basing the number of retransmissions (or the FEC coding redundancy) on the position of the packet within a window, which is central to this paper, has not been suggested before. We emphasize that this idea is generic, and can be incorporated in any sliding-window protocol.

The rest of the paper is structured as follows. Section II describes the model and formally defines the underlying optimization problems. Section III describes basic structural properties of the solution and derives bounds on the optimal strategy performance. Efficient approximation approaches for the 'inner' problem are established in Section IV and incorporated into an overall solution algorithm in Section V. Finally, Section VI concludes with a discussion of our methodology and its possible extensions, and outlines directions for further research.

\section{Model And Problem Formulation}

\section{A. The model}

As explained in the Introduction, we are interested in network connections with a high delay-bandwidth product, in which the receiver accepts packets only in order (with only a small buffer space, if at all, to hold a limited number of out-of-order packets). For our analysis, we shall bring these two characteristics to an extreme. That is, we assume that the receiver is unable to accept out-of-order packets at all, and we take the packet transmission time to be zero, which implies that the size of the window that can be transmitted within a round-trip period is unlimited. Furthermore, we assume there are no other factors that may limit the window size; e.g., the receiving application processes the arriving packets instantly, if necessary, hence no buffer space is consumed by packets arriving in order. These assumptions simplify the analysis and allow it to concentrate on the essential properties of the resulting strategies, without having to deal, from the outset, with details of secondary importance. Section VI discusses the extensions required to alleviate these assumptions, and argues that the solution methodology remains similar nonetheless.

We denote the loss rate in the network by $L$, and assume that losses are independent among packets, as is the case, e.g., for white noise or a random discard policy such as RED [4]. In addition, we neglect the loss rate of acknowledgments, since they are, typically, much shorter than data packets, and therefore suffer less from noise and their paths are often less congested; moreover, since acknowledgments only carry the 
next-expected packet index, a loss of one has no significance if a later one in that window is received successfully. Consequently, for each packet, the sender knows whether it was successfully received after a round-trip time, which we denote by $T$.

We introduce a cost composed of a 'price' of a per unit of time and $b$ per transmitted packet, and define an optimal strategy as one that minimizes the cost/throughput ratio over time; as explained in the Introduction, these prices can have generic interpretations. Incidentally, we chose to base our analysis on this cost structure, which is linear in the time and number of packets, reckoning that it is appropriate for a variety of scenarios and cost interpretations [5]. A different (nonlinear) cost structure may be used instead, provided that the cost of transmitting a window depends only on its size, and not on the identities of its packets or the actual number successfully received. This may affect only the analytical results, e.g. the asymptotic dependence of the optimal strategy performance on the costs, whereas the actual algorithm for finding it remains intact.

The computation of the optimal strategy from the connection parameters $(L, T, a, b)$ implicitly assumes that they are known; therefore, they must either remain constant or change quasi-statically, allowing the strategy to adapt after a change is detected. If any of the parameters, e.g. the round-trip time, changes quickly and unpredictably, it should be modeled by a random variable (e.g., as in [5]) rather than a constant value. We point out, however, that this is not typical of the kind of network connections that are the subject of this study: e.g., for satellite links, the round-trip time is dominated by the propagation delay, which can be considered essentially constant.

The above assumptions readily imply two fundamental properties. First, in the optimal strategy, packets are transmitted only at multiples of $T$; sending packets at other times cannot gain, since no extra information is present. Second, once a sequence of packets is sent at time $t$, the index of the last one to arrive in order is known by time $t+T$, so the strategy simply restarts ('slides') at the subsequent packet. Consequently, the description of a strategy consists simply of a single vector that specifies the number of copies to be sent of every packet, relative to the next-expected index, at every multiple of $T$. The purpose of the subsequent analysis will be to find the optimal such vector.

\section{B. Problem formulation}

Consider a vector $\vec{n}=\left\langle n_{1}, \ldots, n_{i}, \ldots\right\rangle$, where $n_{i}$ are whole and non-negative, and define a random variable $S$ to be the number of in-order successful packets at the receiver if the sender transmits $n_{1}$ repetitions of packet 1 , followed by $n_{2}$ repetitions of packet 2 , etc. ${ }^{\dagger}$ The distribution of $S$ is

$$
P_{S}(j)=\prod_{i=1}^{j}\left(1-L^{n_{i}}\right) \cdot L^{n_{j+1}} .
$$

\footnotetext{
${ }^{\dagger}$ Obviously, transmitting the same packets in any other order can only decrease the expected number of in-order arrivals.
}

We define the score of $\vec{n}$, denoted by $\phi(\vec{n})$, to be the expected value of $S$; thus

$$
\begin{gathered}
\phi(\vec{n}) \triangleq \mathrm{E}[S]=\sum_{j=1}^{\infty} j \cdot \prod_{i=1}^{j}\left(1-L^{n_{i}}\right) \cdot L^{n_{j+1}}= \\
\sum_{j=1}^{\infty} j \cdot\left[\prod_{i=1}^{j}\left(1-L^{n_{i}}\right)-\prod_{i=1}^{j+1}\left(1-L^{n_{i}}\right)\right]= \\
\sum_{j=1}^{\infty} \prod_{i=1}^{j}\left(1-L^{n_{i}}\right) .
\end{gathered}
$$

We seek the vector $\vec{n}=\left\langle n_{1}, \ldots, n_{i}, \ldots\right\rangle$ that minimizes

$$
\frac{a \cdot T+b \cdot \sum_{i=1}^{\infty} n_{i}}{\phi(\vec{n})}=\frac{a \cdot T+b \cdot \sum_{i=1}^{\infty} n_{i}}{\sum_{j=1}^{\infty} \prod_{i=1}^{j}\left(1-L^{n_{i}}\right)} .
$$

The above expression describes the cost/throughput ratio attained by the strategy $\vec{n}$ over time. The numerator is the fixed cost of a period of $T$, during which one window is transmitted, and the denumerator is the expected number of packets successfully communicated in that period.

Consider expression (3) more closely. For any $N$, all the vectors with $\sum_{i=1}^{\infty} n_{i}=N$, i.e. suggesting the same total window size, attain the same numerator value; hence, the comparison among them is based merely on their score. Consequently, let us define

$$
\mathrm{E}_{L}(N) \triangleq \max _{\substack{n_{1}, n_{2}, \ldots \\ \text { s.t. } \sum_{i} n_{i}=N}}\left\{\sum_{j=1}^{\infty} \prod_{i=1}^{j}\left(1-L^{n_{i}}\right)\right\},
$$

and rewrite expression (3) accordingly as

$$
\frac{a \cdot T+b \cdot N}{\mathrm{E}_{L}(N)} \text {. }
$$

Then, the problem of finding the strategy vector that minimizes (5) can be separated into the following (sub-)problems:

Inner problem: Computing $\mathrm{E}_{L}(N)$ for a given $N$. Outer problem: Searching for $N^{*}$ that minimizes (5).

This separation is convenient in that it isolates the infinitedimensional part of the problem to depend solely on $L$, while the dependence on the other parameters reduces to a onedimensional optimization only. Furthermore, the vector that actually attains the maximum in (4) is not needed until the final stage, after $N^{*}$ has been found; during the search of $N$, it suffices to be able to evaluate $\mathrm{E}_{L}(N)$, without the need to find the maximizing vector explicitly.

To conclude this section, we digress to consider the case of 'classic' sliding windows, where each packet is sent only once in a window; this corresponds to the vector $n_{1}=\cdots=$ $n_{N}=1$, with a cost/throughput ratio of

$$
\frac{a \cdot T+b \cdot N}{\sum_{j=1}^{N}(1-L)^{j}}=\frac{L}{1-L} \cdot \frac{a \cdot T+b \cdot N}{1-(1-L)^{N}} .
$$


Maximizing this (e.g. by differentiating with respect to $N$ ) yields an optimal window size of

$$
\begin{aligned}
N^{*} & =\frac{1}{\log \frac{1}{1-L}}\left[-\operatorname{plog}\left(-\frac{(1-L)^{\frac{a T}{b}}}{e}\right)-1+\right. \\
& \left.\frac{a T}{b} \log (1-L)\right] \underset{(\text { if } a T \gg b)}{\approx} \frac{\log \left(\frac{a T}{b} \log \frac{1}{1-L}+1\right)}{\log \frac{1}{1-L}},
\end{aligned}
$$

where $\operatorname{plog}(\cdot)$ (the product-log function) denotes the inverse function of $f(t)=t \cdot e^{t}$, such that $t=-\operatorname{plog}(-y)$ (for $\left.0<y \leq \frac{1}{e}\right)$ is the largest positive solution to the equation $y=t \cdot e^{-t}$; in the final approximation we used the property that $-\operatorname{plog}\left(-e^{-x}\right) \approx x+\log x$ for $x \gg 1 .^{\dagger}$ Thus, as the time cost $a$ increases with respect to the other parameters, the optimal window size increases logarithmically in $a$. Since the denominator of (6) tends to a finite value as $N \rightarrow \infty$, the cost/throughput ratio, overall, increases linearly in $a$.

\section{BASIC PROPERTIES AND BOUNDS}

In this section, we show some basic structural properties of the optimization problems' solutions, and derive important bounds, in particular, on their asymptotic behavior.

\section{A. Properties of the inner problem}

Our first two lemmas state basic and intuitively obvious structural properties.

Lemma 1. $\mathrm{E}_{L}(N)$ decreases in $L$ and increases in $N$.

Proof. Consider the maximizing vector in (4) for some $L$ and $N$, and suppose that $L$ is then decreased. The score of that vector then increases; if it is no longer the maximizer for the new $L$, then, obviously, the maximum value can only be even higher. Therefore, the value of (4) increases.

Alternatively, suppose that $N$ is increased, and add the entire amount of the increase to the first element (arbitrarily). Again, this results in an increase of the score; if the resulting vector is not the maximizer for the new $N$, the value of (4) can only increase further.

Lemma 2. For a given $N$, the elements of the vector that achieves the maximum in (4) maintain a non-increasing order, i.e. $n_{1} \geq n_{2} \geq \cdots \geq n_{i} \geq \ldots$.

Proof. Suppose, by contradiction, that there exists a pair of indices $i_{1}<i_{2}$ with $n_{i_{1}}<n_{i_{2}}$. Consider the score of the vector resulting by swapping $n_{i_{1}}, n_{i_{2}}$, as given by expression (2). All the sum elements (products) for $j<i_{1}$ (which depend on neither $n_{i_{1}}$ nor $n_{i_{2}}$ ), as well as for $j \geq i_{2}$ (which contain both $\left(1-L^{n_{i_{1}}}\right)$ and $\left(1-L^{n_{i_{2}}}\right)$ in the product), remain unchanged. The elements for $i_{1} \leq j<i_{2}$, which contain only $\left(1-L^{n_{i_{1}}}\right)$ but not $\left(1-L^{n_{i_{2}}}\right)$ in the product, are strictly increased by the swap, thereby increasing the value of the entire sum. Consequently, the original vector cannot be a maximizer.

\footnotetext{
${ }^{\dagger}$ The product-log function is also known elsewhere as Lambert's Wfunction [14], or, more precisely, as one of its real-valued branches.
}

Corollary 1. In the maximizing vector, all the elements after the first zero element are also zero.

Corollary 2. For a given $N$, the index of the last nonzero element in the maximizing vector is bounded by $N$.

We proceed to derive an important bound on the number of transmissions required to attain a given score. For this purpose, we introduce a variable change that makes the subsequent presentation more convenient. Define $p_{i} \triangleq 1-L^{n_{i}}$ (i.e. $p_{i}$ is the individual probability of packet $i$ to arrive successfully, regardless of other packets). We shall refer to the vector $\vec{p}=$ $\left\langle p_{1}, \ldots, p_{i}, \ldots\right\rangle$ as completely equivalent to the vector $\vec{n}$ and interchange them freely for convenience; in particular, with a slight abuse of notation, we refer to $\phi(\vec{p})=\sum_{j=1}^{\infty} \prod_{i=1}^{j} p_{i}$ as the score of $\vec{p}$.

Lemma 3. If $\vec{n}$ is the maximizing vector in (4), then $p_{1}=$ $1-L^{n_{1}} \geq \frac{\phi(\vec{n})}{\phi(\vec{n})+1}$.

Proof. Lemma 2 implies that $p_{i} \leq p_{1}$ for all $i$; therefore,

$$
\phi(\vec{n})=\sum_{j=1}^{\infty} \prod_{i=1}^{j} p_{i} \leq \sum_{j=1}^{\infty}\left(p_{1}\right)^{j}=\frac{p_{1}}{1-p_{1}}
$$

and the lemma immediately follows by extracting $p_{1}$.

Theorem 1. For any vector $\vec{n}, \quad N=\sum_{i} n_{i} \geq$ $\log _{1 / L}\{[\phi(\vec{n})+1] !\} .^{\ddagger}$

Proof. Obviously, since the factorial and the logarithm are monotonously increasing operations, it suffices to prove the theorem for the vector with the maximum score for a given $N$. Such a vector must satisfy lemmas $2-3$.

Consider the equivalent vector $\vec{p}=\left\langle p_{1}, \ldots, p_{M}, 0, \ldots\right\rangle$, where $M$ denotes the index of the last non-zero element. Define the following sequence of subvectors, $\vec{p}^{(m)} \triangleq$ $\left\langle p_{m}, p_{m+1}, \ldots, p_{M}, 0,0, \ldots\right\rangle$, and of their corresponding scores, $\phi_{m}=\phi\left(\vec{p}^{(m)}\right)=\sum_{j=m}^{M} \prod_{i=m}^{j} p_{i}$, for all $1 \leq m \leq$ $M$; note that $\phi_{1}$ is the score of the original vector. Observe that $\phi_{m}=p_{m}\left(1+\phi_{m+1}\right)$, and, therefore, $\phi_{m+1} \geq \phi_{m}-1$, for all $1 \leq m<M$; successively applying this inequality, we get $\phi_{m} \geq \phi_{1}-(m-1)$ for all $m$. On the other hand, applying Lemma 3 on each of the subvectors in turn, we have $p_{m} \geq \frac{\phi_{m}}{\phi_{m}+1}$, or $\frac{1}{1-p_{m}} \geq \phi_{m}+1$. Consequently,

$$
\begin{aligned}
{\left[\prod_{m=1}^{M}\left(1-p_{m}\right)\right]^{-1} \geq } & \prod_{m=1}^{M}\left(\phi_{m}+1\right) \geq \\
& \prod_{m=1}^{M} \max \left[\phi_{1}-(m-1)+1,1\right] .
\end{aligned}
$$

Now, consider the factorial $\left(\phi_{1}+1\right)$ !. Denote $\left\lfloor\phi_{1}\right\rfloor$ to be the integer part of $\phi_{1}$ (and, thereby, $\left(\phi_{1}-\left\lfloor\phi_{1}\right\rfloor\right)$ to be its

\footnotetext{
${ }^{\ddagger}$ Recall that the factorial $t$ !, for any $t \geq 0$, is defined by $t$ ! $=$ $\int_{0}^{\infty} x^{t} e^{-x} d x$; this definition conincides with the more common $t !=1 \cdot 2$. $\ldots \cdot t$ for integer $t$. A well-known property of the factorial is $t !=t \cdot(t-1)$ ! for any $t \geq 1$.
} 
fractional part). Successively applying the factorial property of $t !=t \cdot(t-1)$ ! for any $t \geq 1$, we have

$$
\begin{gathered}
\left(\phi_{1}+1\right) !=\left(\phi_{1}+1\right) \cdot \phi_{1} \cdot\left(\phi_{1}-1\right) \cdot \ldots \cdot\left(\phi_{1}-\left\lfloor\phi_{1}\right\rfloor\right) != \\
\prod_{m=1}^{M} \max \left[\phi_{1}-(m-1)+1,1\right] \cdot\left(\phi_{1}-\left\lfloor\phi_{1}\right\rfloor\right) ! \leq \\
\prod_{m=1}^{M} \max \left[\phi_{1}-(m-1)+1,1\right] . \quad(10)
\end{gathered}
$$

Note that we implicitly used the obvious fact that $\phi_{1} \leq M$, and also that $t ! \leq 1$ for any $0 \leq t<1$.

Combining inequalities (9) and (10), we obtain $\left[\prod_{m}\left(1-p_{m}\right)\right]^{-1} \geq\left(\phi_{1}+1\right)$ !. Taking the logarithm of both sides and noting that $\log _{1 / L}\left(1-p_{m}\right)=-n_{m}$, we finally get $\sum_{m} n_{m} \geq \log _{1 / L}\left[\left(\phi_{1}+1\right) !\right]$.

Finally, the following fundamental theorem presents the asymptotic relation between the window size and the maximum score that can be obtained by a vector of that size.

Theorem 2. $\mathrm{E}_{L}(N)=\Theta\left(\frac{N}{\log _{1 / L} N}\right){ }^{\dagger}$

Proof. We apply the well-known Stirling's factorial approximation formula, $t ! \approx \sqrt{2 \pi t}\left(\frac{t}{e}\right)^{t}$ for large $t$, to the inequality established in Theorem 1, and obtain

$$
\begin{array}{r}
N \geq \log _{1 / L}\left[\mathrm{E}_{L}(N)+1\right] ! \approx \log _{1 / L}\left(\frac{\mathrm{E}_{L}(N)+1}{e}\right) . \\
{\left[\mathrm{E}_{L}(N)+1\right]+\log _{1 / L} \sqrt{2 \pi\left[\mathrm{E}_{L}(N)+1\right]} ;}
\end{array}
$$

thus, $N=\Omega\left(\mathrm{E}_{L}(N) \cdot \log _{1 / L} \mathrm{E}_{L}(N)\right)$. This implies directly that $\mathrm{E}_{L}(N)=O\left(\frac{N}{\log _{1 / L} N}\right)$.

To show that $\mathrm{E}_{L}(N)=\Omega\left(\frac{N}{\log _{1 / L} N}\right)$ as well, it suffices to find one example of a vector that attains a score of $\Omega\left(\frac{N}{\log _{1 / L} N}\right)$. Accordingly, consider the vector $\left\langle n_{1}, \ldots, n_{M}, 0, \ldots\right\rangle$, such that $n_{1}=\cdots=n_{M}=\log _{1 / L} N$ and $M=\frac{N}{\log _{1 / L} N}$. Its score is

$$
\begin{aligned}
& \sum_{j=1}^{M} \prod_{i=1}^{j}\left(1-L^{n_{i}}\right)= \sum_{j=1}^{\frac{N}{\log _{1 / L} N}}\left(1-L^{\log _{1 / L} N}\right)^{j}= \\
& N\left(1-\frac{1}{N}\right) \cdot\left[1-\left(1-\frac{1}{N}\right)^{\frac{N}{\log _{1 / L} N}}\right] \geq \\
& N\left(1-\frac{1}{N}\right)\left(1-e^{-\frac{1}{\log _{1 / L} N}}\right),
\end{aligned}
$$

completing the proof, as $e^{-\frac{1}{x}} \approx 1-\frac{1}{x}$ for large $x .^{\ddagger}$

\footnotetext{
${ }^{\dagger}$ Recall that $f(N)=O(g(N))$, for positive functions $f(N), g(N)$, means that $\lim _{N \rightarrow \infty} \frac{f(N)}{g(N)}<\infty$; in addition, $f(N)=\Omega(g(N))$ is equivalent to $g(N)=O(f(N))$, and $f(N)=\Theta(g(N))$ means that both $f(N)=$ $O(g(N))$ and $f(N)=\Omega(g(N))$.

$\ddagger_{\text {The fact that }} \log _{1 / L} N$ and/or $\frac{N}{\log _{1 / L} N}$ may not be integers is insignificant: rounding both expressions up to the nearest integers only increases the vector's score further, with an asymptotically negligible impact on the window size.
}

It is insightful to compare the result of Theorem 2 with the total number of packets received successfully (not necessarily in order), which is, obviously, $N \cdot(1-L)$, i.e., $\Theta(N)$. Hence, it can be said that discarding out-of-order packets impacts the performance by a logarithmic factor. This theorem can also be used inversely: in order to have an expected number of $\phi$ packets arriving successfully and in-order to the destination, the total number of packet copies transmitted by the source must be $\Theta\left(\phi \cdot \log _{1 / L} \phi\right)$.

\section{B. Properties of the outer problem}

This subsection is concerned with the dependence of the optimal window size $N^{*}$ on the cost factors $a, b$. Theorem 3 states an intuitively evident monotonicity property. Theorem 4 presents the central result of this section, regarding the asymptotic dependence of the cost/throughput ratio on the time cost $a$.

Theorem 3. The optimal $N^{*}$ is non-decreasing in $\frac{a T}{b}$.

Proof. Consider two sets of parameters $a_{1}, b_{1}, T_{1}$ and $a_{2}, b_{2}, T_{2}$ such that $\frac{a_{1} T_{1}}{b_{1}} \geq \frac{a_{2} T_{2}}{b_{2}}$, and suppose that $N_{1}^{*}, N_{2}^{*}$ are their corresponding solutions (to the outer problem). This implies, in particular, that

$$
\begin{aligned}
& \frac{\frac{a_{1} T_{1}}{b_{1}}+N_{2}^{*}}{\mathrm{E}_{L}\left(N_{2}^{*}\right)} \geq \frac{\frac{a_{1} T_{1}}{b_{1}}+N_{1}^{*}}{\mathrm{E}_{L}\left(N_{1}^{*}\right)}, \\
& \frac{\frac{a_{2} T_{2}}{b_{2}}+N_{2}^{*}}{\mathrm{E}_{L}\left(N_{2}^{*}\right)} \leq \frac{\frac{a_{2} T_{2}}{b_{2}}+N_{1}^{*}}{\mathrm{E}_{L}\left(N_{1}^{*}\right)} .
\end{aligned}
$$

Subtracting the second inequality from the first and noting that the common factor $\left(\frac{a_{1} T_{1}}{b_{1}}-\frac{a_{2} T_{2}}{b_{2}}\right)$ is positive, we obtain $\mathrm{E}_{L}\left(N_{1}^{*}\right) \geq \mathrm{E}_{L}\left(N_{2}^{*}\right)$. In light of the monotonicity of $\mathrm{E}_{L}(N)$ (Lemma 1), this implies $N_{1}^{*} \geq N_{2}^{*}$.

Theorem 4. As $a \rightarrow \infty$ (for fixed values of $T, b, L$ ), the cost/performance ratio attained by the optimal strategy increases logarithmically in a.

Proof. Consider the expression $h(x) \triangleq \frac{a \cdot T+b \cdot x}{x / \log _{1 / L} x}$, as a function of a (continuous) variable $x$. By differentiation with respect to $x$, it is easily found that its minimum is attained at $x^{*}=\frac{a T}{b} \cdot\left[-\operatorname{plog}\left(-e \cdot \frac{b}{a T}\right)\right] .{ }^{\S}$ Using again the property that $-\operatorname{plog}\left(-e^{-y}\right) \approx y+\log y$ for large $y$, we obtain $x^{*}=\Theta(a$. $\log a)$, and the minimum value of $h(x)$ is therefore $\Theta(\log a)$. This proves the theorem, since, in light of Theorem 2, the cost/throughput ratio is itself $\Theta(h(N))$, and its minimum value can, therefore, deviate from that of $h(N)$ by a constant factor at most.

Thus, the ability to use retransmissions within the window enables the average cost per successful packet to increase merely logarithmically in $a$, rather than linearly as in the case of 'classic' sliding windows. Incidentally, note that no similar result exists for $b \rightarrow \infty$ with the other parameters constant; indeed, as $\frac{a T}{b} \rightarrow 0$, the optimal strategy tends to $\langle 1,0,0, \ldots\rangle$ (simple stop-and-wait), and the value of expression (5) simply

\footnotetext{
${ }^{\S}$ Recall the definition of the plog function at the end of Section II.
} 
Initialization: Set $\vec{n}=\langle 0,0, \ldots\rangle$

Do $N$ times:

For every $j$ such that either $j=1$ or $n_{j-1}>n_{j}$ :

Temporarily set $n_{j} \leftarrow n_{j}+1$

Compute the score of $\vec{n}$

Restore $n_{j}$

For the $j$ that got the best score: Set $n_{j} \leftarrow n_{j}+1$

Fig. 1. Algorithm Greedy-Inner (GI)

increases linearly in $b$. This is true, of course, for the 'classic' case as well.

\section{Solution of the InNer Problem}

The function $\mathrm{E}_{L}(N)$ can be computed directly by exhaustive search among vectors with nonnegative integer elements that sum up to $N$. In light of Lemma 2, it suffices to limit the search to vectors in which the elements maintain a nonincreasing order. This reduces the search space considerably; e.g., for $N=10$ there are only 42 vectors to check, and the number increases to 204226 for $N=50$ and 190569292 for $N=100$. Hence, this approach is quite viable for relatively small $N$.

For larger $N$, however, exhaustive search may be impractical, and we seek alternatives to yield reasonable approximations at a low computational cost. Subsection A presents a 'greedy' direct-search algorithm; then subsection B presents a solution method for a similar auxiliary problem in continuous variables. The two approaches are evaluated and compared in subsection $\mathrm{C}$.

\section{A. Greedy search}

Our first approach for approximation of $\mathrm{E}_{L}(N)$ is a direct search algorithm, shown in Figure 1, which we term GI (for "Greedy Inner"). It begins with a vector of all-zeros and performs $N$ iterations, incrementing one element each time specifically, the one whose increment brings about the highest score in that iteration. The following example demonstrates the algorithm results as opposed to exhaustive search; a more detailed discussion of the algorithm's performance appears in subsection $\mathrm{C}^{\dagger}$

Example: The following table summarizes the optimal vectors and their scores for $N=15$ and selected values of $L$.

\begin{tabular}{|c|c|c|}
\hline$L$ & Optimal vector & Score \\
\hline 0.1 & $\langle 2,2,2,2,1,1,1,1,1,1,1,0,0, \ldots\rangle$ & 8.41131 \\
0.3 & $\langle 3,2,2,2,2,2,1,1,0,0, \ldots\rangle$ & 5.39436 \\
0.5 & $\langle 4,3,3,2,2,1,0,0, \ldots\rangle$ & 3.61954 \\
0.7 & $\langle 6,5,3,1,0,0, \ldots\rangle$ & 2.24336 \\
0.9 & $\langle 11,4,0,0, \ldots\rangle$ & 0.92217 \\
\hline
\end{tabular}

In accordance with intuition, for low loss rates, it is best to send at least one copy of more individual packets; conversely, when

\footnotetext{
†Other greedy algorithms can be devised based on moves instead of increments; e.g., one may start with $\langle N, 0,0, \ldots\rangle$ and seek score improvements by "moving to the right", or start with $\langle 1, \ldots, 1,0,0, \ldots\rangle$ and "move to the $\underbrace{1, \ldots}_{N}$

left". In [15], such algorithms are shown to result in occasional and usually insignificant improvements over GI, while possessing a complexity that is higher by a factor of $N$ at least.
}

the loss rate is high, the expected number of successful in-order arrivals is maximized by duplicating just the first few packets. In fact, it is obvious that, for any $N$, the optimal vector tends to $\langle\underbrace{1, \ldots, 1}_{N}, 0,0, \ldots\rangle$ for $L \rightarrow 0$ and to $\langle N, 0,0, \ldots\rangle$ for $L \rightarrow 1$.

The values in the above table were found by exhaustive search. Algorithm GI does not converge to these vectors in all cases; specifically, for $L=0.3$, it finds the vector $\langle 3,3,2,2,2,2,1,0,0, \ldots\rangle$, with a somewhat lower score of 5.38234 , and for $L=0.5$, it converges to $\langle 4,4,3,3,1,0,0, \ldots\rangle$, with a score of 3.59482 .

\section{B. Approximation through continuous relaxation}

We now analyze the properties of the optimization problem that defines the function $\mathrm{E}_{L}(N)$, expressed by (4), omitting the requirement for the elements of $\vec{n}$ to be integers. This way, we have a relaxed optimization problem in a continuous space, which can be analyzed more easily by 'traditional' methods from optimization theory. Obviously, this technique results in a value that is higher than $\mathrm{E}_{L}(N)$ (unlike directsearch algorithms such as GI, which result in vectors with lower scores than $\mathrm{E}_{L}(N)$ ).

To distinguish the relaxed problem from the original one, we denote the maximum score by $\Phi_{L}(s)$, where $s$ (rather than $N$ ) is used to denote the vector size, to emphasize that $\Phi_{L}(\cdot)$, unlike $\mathrm{E}_{L}(\cdot)$, is well-defined for non-integer arguments. Additionally, we again make the convenient variable change of $p_{i} \triangleq 1-L^{n_{i}}$, after which the score expression is simply $\sum_{j=1}^{\infty} \prod_{i=1}^{j} p_{i}$, while the constraints on the vector elements become

$$
\begin{aligned}
n_{i} & \geq 0 \Longrightarrow 0 \leq p_{i} \leq 1 ; \\
\sum_{i} n_{i} & =s \Longrightarrow \prod_{i}\left(1-p_{i}\right)=L^{s} .
\end{aligned}
$$

We immediately observe that the problem essentially depends on just one parameter, $L^{s}$, i.e. $\Phi_{L}(s)=\Phi\left(L^{s}\right)$ (even though the translation back to the original variables, $n_{i}=$ $\log _{L}\left(1-p_{i}\right)$, involves the specific value of $\left.L\right)$.

Obviously, the solution vector, which attains the globally maximum score, must, in particular, be also a local maximum; as both the target expression and the constraint are differentiable, this means that it has to satisfy the corresponding Kuhn-Tucker conditions [16], [15]. These can be verified, by a straightforward simplification, to require that there exists a constant $\lambda$ such that

$$
\begin{aligned}
&\left(1-p_{m}\right) \sum_{j=m}^{\infty} \prod_{\substack{i=1 \\
i \neq m}}^{j} p_{i}=\lambda \quad\left(\text { if } 0<p_{m}<1\right), \\
& \sum_{j=m}^{\infty} \prod_{\substack{i=1 \\
i \neq m}}^{j} p_{i} \leq \lambda \quad\left(\text { if } p_{m}=0\right) . \ddagger
\end{aligned}
$$

Using (15)-(16), we can prove the following claim about the structure of the solution vector.

\footnotetext{
${ }^{\ddagger}$ In principle, there should also be a condition for $p_{m}=1$; however, it is immediately seen that $p_{m}=1$ for any $m$ contradicts constraint (14)
} 
Lemma 4. A vector that satisfies conditions (13)-(16) has the form $\left\langle p_{1}, p_{2}, \ldots, p_{M}, 0,0, \ldots\right\rangle$, where $M$ is finite, $\left.\left.p_{1}\right\rangle \cdots\right\rangle$ $p_{M}>0$, and $p_{M} \leq \frac{1}{2}$.

Proof. First, note that if $p_{k}=0$ for some $k$, then condition (15) cannot be satisfied for any $m>k$, since all the products contain $p_{k}$ and hence equal 0 . Therefore, there are no positive elements after the first zero element.

For any $1 \leq m<M$, condition (15) implies that

$$
\frac{1-p_{m}}{p_{m}} \sum_{j=m}^{\infty} \prod_{i=1}^{j} p_{i}=\frac{1-p_{m+1}}{p_{m+1}} \sum_{j=m+1}^{\infty} \prod_{i=1}^{j} p_{i} .
$$

Since, obviously, $\sum_{j=m}^{\infty} \prod_{i=1}^{j} p_{i}>\sum_{j=m+1}^{\infty} \prod_{i=1}^{j} p_{i}$, it follows that $\frac{1-p_{m}}{p_{m}}<\frac{1-p_{m+1}}{p_{m+1}}$, which implies $p_{m}>p_{m+1}$.

Comparing expression (16) for $p_{M+1}$ with expression (15) for $p_{M}$, we have

$$
\prod_{i=1}^{M} p_{i} \leq\left(1-p_{M}\right) \prod_{i=1}^{M-1} p_{i}
$$

and after dividing both sides by the common factor of $\prod_{i=1}^{M-1} p_{i}$, it reduces to $p_{M} \leq 1-p_{M}$, hence $p_{M} \leq \frac{1}{2}$.

It remains to show that $M$ is finite, i.e., the vector cannot have infinitely many positive elements. Suppose, by contradiction, that such a vector exists. Then constraint (14) implies that $p_{k} \underset{k \rightarrow \infty}{\longrightarrow} 0$. Consequently, choose an index $K$ such that $p_{K}<\frac{1}{2}$. We now show that the assumption $p_{K+1}>0$ leads to a contradiction.

If $p_{K+1}>0$, then condition (15) implies

$$
\frac{1-p_{K+1}}{p_{K+1}} \sum_{j=K+1}^{\infty} \prod_{i=1}^{j} p_{i}=\frac{1-p_{K}}{p_{K}} \sum_{j=K}^{\infty} \prod_{i=1}^{j} p_{i},
$$

or, dividing both sides by the common factor of $\prod_{i=1}^{K} p_{i}$,

$$
\begin{aligned}
& \frac{1-p_{K+1}}{p_{K+1}} \sum_{j=K+1}^{\infty} \prod_{i=K+1}^{j} p_{i}= \\
& \frac{1-p_{K}}{p_{K}} \cdot\left(1+\sum_{j=K+1}^{\infty} \prod_{i=K+1}^{j} p_{i}\right),
\end{aligned}
$$

therefore

$$
\sum_{j=K+1}^{\infty} \prod_{i=K+1}^{j} p_{i}=\frac{\left(1-p_{K}\right) p_{K+1}}{p_{K}-p_{K+1}}
$$

However, we showed above that the positive elements of $\vec{p}$ must maintain a decreasing order; hence, $p_{i}<p_{K+1}$ for $i>$ $K+1$, and the following inequality holds:

$$
\sum_{j=K+1}^{\infty} \prod_{i=K+1}^{j} p_{i} \leq \sum_{j=K+1}^{\infty}\left(p_{K+1}\right)^{j-K}=\frac{p_{K+1}}{1-p_{K+1}} .
$$

Substituting this inequality into (21), we get

$$
\frac{1-p_{K}}{p_{K}-p_{K+1}} \leq \frac{1}{1-p_{K+1}} \Longrightarrow 1+p_{K} p_{K+1} \leq 2 p_{K},
$$

which contradicts $p_{K}<\frac{1}{2}$. Thus, condition (15) cannot be satisfied; therefore, $p_{K+1}=0$.

Suppose that a vector $\left\langle p_{1}, p_{2}, \ldots, p_{M}, 0,0, \ldots\right\rangle$ is known to satisfy conditions (13) and (15)-(16), and that only the value of the last element $p_{M}$ is known. Then the other elements can be uniquely determined by a procedure of backward iteration. Specifically, once the values of $p_{m+1}, \ldots, p_{M}$ are known, one can equate expression (15) for $p_{m}$ and $p_{m+1}$, divide by the common factor of $\prod_{i=1}^{m-1} p_{i}$, and extract $p_{m}$. This results in the formula

$$
p_{m}=\frac{1+\sum_{j=m+1}^{M} \prod_{i=m+1}^{j} p_{i}}{2+\sum_{j=m+2}^{M} \prod_{i=m+2}^{j} p_{i}} .
$$

Conversely, it is easy to see that, for any $M$ and $0<$ $p_{M} \leq \frac{1}{2}$, the vector obtained through formula (24) satisfies conditions (15)-(16). For convenience, we define a function $\overrightarrow{\boldsymbol{p}}: \mathbb{R}^{+} \longmapsto \mathbb{R}^{\infty}$, such that, for any $t>0, \overrightarrow{\boldsymbol{p}}(t)$ is the vector corresponding to $M=\lceil t\rceil^{\dagger}$ and $p_{M}=\frac{1}{2}(t+1-\lceil t\rceil)$. This puts the set of all vectors that satisfy conditions (15)-(16) (and are therefore "eligible candidates" to be solutions to the optimization problem for the corresponding values of $L^{s}$ ) in one-to-one correspondence with the positive real axis. We also define $\boldsymbol{p}_{i}: \mathbb{R}^{+} \longmapsto \mathbb{R}$ to be the $i$-th component of $\overrightarrow{\boldsymbol{p}}$.

\section{Lemma 5. The function $\overrightarrow{\boldsymbol{p}}$ is continuous.}

Proof. The continuity of $\vec{p}$ at non-integer points (continuity in $p_{M}$ only, for a fixed $M$ ) is obvious from formula (24), which shows $p_{m}$, for any $1 \leq m \leq M-1$, to be continuous in $p_{m+1}, \ldots, p_{M}$, and therefore (applying backward induction from $m=M-1$ to $m=1$ ) to be continuous in $p_{M}$.

To show the continuity of $\overrightarrow{\boldsymbol{p}}$ at $t=K$ for an integer $K$, one must prove $\lim _{t \rightarrow K^{-}} \overrightarrow{\boldsymbol{p}}(t)=\lim _{t \rightarrow K^{+}} \overrightarrow{\boldsymbol{p}}(t)$. Consider first the component $\boldsymbol{p}_{K}$. For $t \rightarrow K^{-}, \boldsymbol{p}_{K}(t)$ is simply the last nonzero element of $\overrightarrow{\boldsymbol{p}}(t)$; that is, $M=K$ and $\boldsymbol{p}_{K}(t)=\frac{1}{2}(t+1-K)$. Therefore,

$$
\lim _{t \rightarrow K^{-}} \boldsymbol{p}_{K}(t)=\lim _{t \rightarrow K^{-}} \frac{1}{2}(t+1-K)=\frac{1}{2} .
$$

For $t \rightarrow K^{+}, \boldsymbol{p}_{K}(t)$ is the penultimate nonzero element; that is, $M=K+1, p_{M}=\frac{1}{2}(t-K)$, and $\boldsymbol{p}_{K}(t)$ can be computed from (24):

$$
\lim _{t \rightarrow K^{+}} \boldsymbol{p}_{K}(t)=\lim _{t \rightarrow K^{+}} \frac{1+\frac{1}{2}(t-K)}{2}=\frac{1}{2} .
$$

Hence, the component $\boldsymbol{p}_{K}(t)$ is continuous at $t=K$. From here, the continuity of $\boldsymbol{p}_{1}(t), \ldots, \boldsymbol{p}_{K-1}(t)$ in $t$ follows from their continuity in $\boldsymbol{p}_{K}$, according to (24) (again, using backward induction from $m=K-1$ to $m=1$ ).

Figure 2 shows plots of a few functions derived from the definition of $\overrightarrow{\boldsymbol{p}}(t)$. Figure 2-a shows a plot of $\boldsymbol{p}_{1}(t)$, using a logarithmic vertical axis to emphasize the 'waviness' of the function. Note that, by construction, $\boldsymbol{p}_{i}(t)=\boldsymbol{p}_{i+k}(t+k)$ for

\footnotetext{
$\dagger$ The operator $\lceil t\rceil$ denotes the integer obtained by rounding up of $t$, i.e. the smallest integer that is not less than $t$.
} 


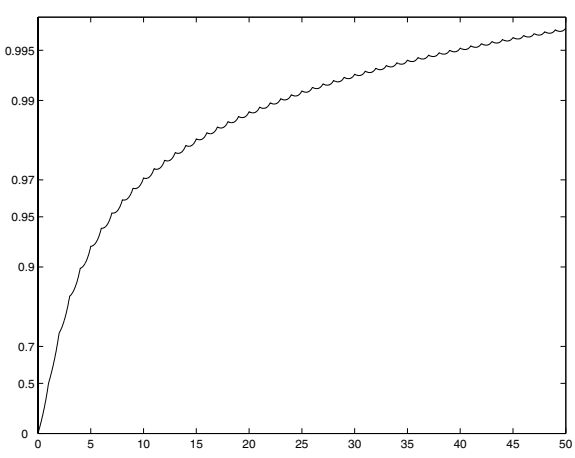

Fig. 2-a. Plot of $\boldsymbol{p}_{1}(t)$.

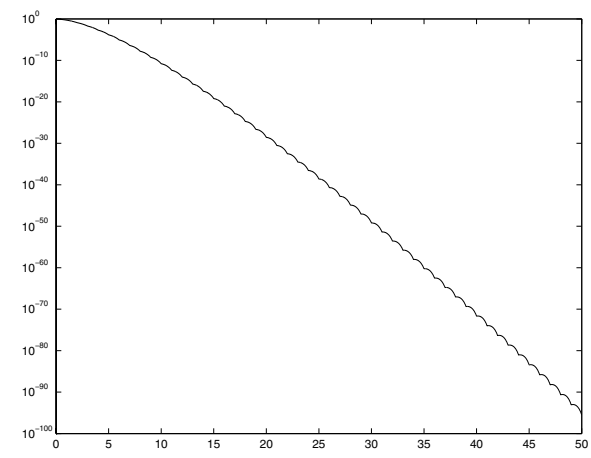

Fig. 2-b. Plot of $L^{s}(t) \triangleq \prod_{i}\left(1-\boldsymbol{p}_{i}(t)\right)$.

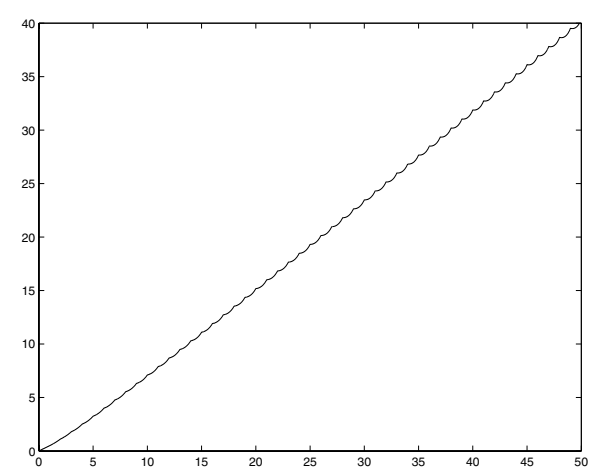

Fig. 2-c. Plot of $\phi(t) \triangleq \sum_{j=1}^{\infty} \prod_{i=1}^{j} \boldsymbol{p}_{i}(t)$. any integer $k$ and any $t>0$; hence, appropriately shifted, the plot is valid for any component $\boldsymbol{p}_{m}(t)$. Figure 2-b shows a plot of $L^{s}(t) \triangleq \prod_{i=1}^{\infty}\left(1-\boldsymbol{p}_{i}(t)\right)$, i.e. the value of $L^{s}$ for which the vector $\overrightarrow{\boldsymbol{p}}(t)$ would satisfy constraint (14); for convenience, the vertical axis is logarithmic here as well. Finally, Figure 2-c shows a plot of the score attained by $\overrightarrow{\boldsymbol{p}}(t)$.

Conceptually, the solution of the optimization problem for a given $L^{s}$ is obtained by locating the set of points $\left\{t \mid \prod_{i}\left[1-\boldsymbol{p}_{i}(t)\right]=L^{s}\right\}$ (e.g., from Figure 2-b), and selecting the point that attains the maximal value of $\sum_{j} \prod_{i=1}^{j} \boldsymbol{p}_{i}(t)$ (e.g., from Figure 2-c). Note that the set contains more than one point for $L^{s} \lesssim 1.586 \cdot 10^{-43}$ (the function of Figure 2-b ceases to be strictly decreasing after $t=27$ ). An algorithm to compute the solution could begin by evaluating $L^{s}(t)$ at integer points, exploiting the function's continuity to find an initial search range, and then perform a detailed search, e.g., by evaluation of $L^{s}(t)$ on a sufficiently dense grid of points (depending on the required precision) and subsequent interpolation. The implementation details of such an algorithm are tedious yet entirely straightforward, and are not considered here further.

Our experience from running this computation for various problem instances suggests that different values of $t$ that correspond to the same $L^{s}$ tend to attain very close values of $\phi$ as well, hence simply finding any such $t$ is nearly optimal. In graphical terms, this means that the plots in figures 2-b and 2-c are very nearly "mirror images" of each other (and become ever more so as $t$ gets larger). To illustrate this, Figure 3 shows a parametric plot of $\phi(t)$ versus $L^{s}(t)$. Observe that the plot is virtually indistinguishable from a function; it takes a great deal of "zooming in" to notice that the plot actually zig-zags back and forth, and that every $L^{s} \lesssim 1.586 \cdot 10^{-43}$ has several corresponding values of $\phi$, of which only the topmost one is the 'true' $\Phi\left(L^{s}\right)$. Thus, strictly speaking, the function $\Phi\left(L^{s}\right)$ is not continuous; however, its 'jumps' are markedly minuscule.

Incidentally, it can be observed that the proof of Theorem 1 is easily extended to the continuous version of the problem; it then states that $L^{s} \leq \frac{1}{\left[\Phi\left(L^{s}\right)+1\right] !}$. This bound is plotted by the dotted line in Figure 3. Thus, it can be seen that the auxiliary function $\Phi\left(L^{s}\right)$, while not difficult to compute, provides a

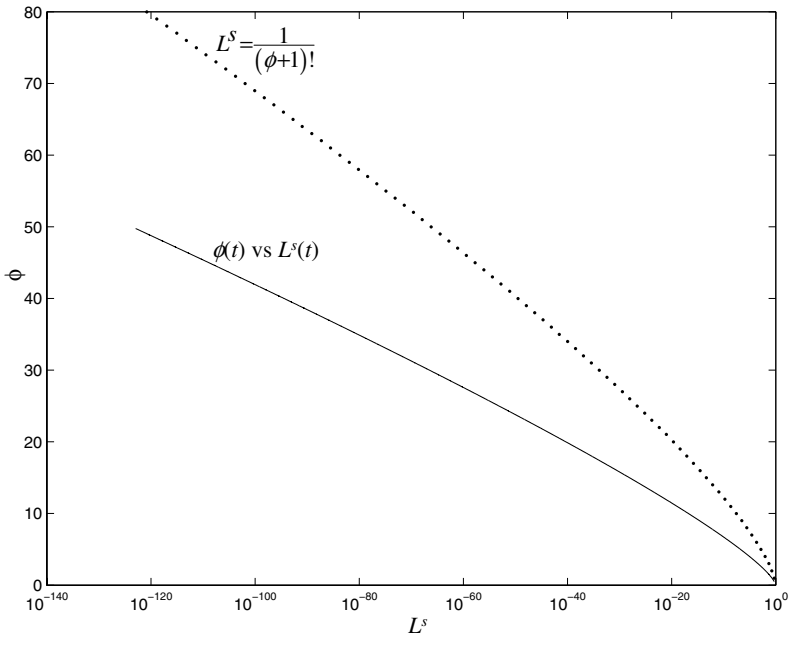

Fig. 3. Parametric plot of $\phi(t)$ vs $L^{s}(t)$, and comparison to the bound implied by Theorem 1 .

much tighter bound.

\section{Evaluation of the approaches}

We begin with a theorem that provides the asymptotic connection between $\mathrm{E}_{L}(N)$ and the auxiliary function $\Phi_{L}(s)$. It states that, in a certain sense, $\Phi_{L}(s)$ closely approximates $\mathrm{E}_{L}(N)$ for large values of $N$.

Theorem 5. For any $L, s, \mathrm{E}_{\left(L^{s}\right)^{1 / N}}(N) \underset{N \rightarrow \infty}{\longrightarrow} \Phi_{L}(s)$.

Proof. Define the following auxiliary function, for $0<\Lambda<1$ and $0 \leq p<1: Y_{\Lambda}(p)=1-\Lambda^{\left\lfloor\log _{\Lambda}(1-p)\right\rfloor}$, where $\lfloor\cdot\rfloor$ denotes the integer-part operator. Thus, $Y_{\Lambda}(p)$ is the highest number that is no higher than $p$ and can be expressed as $1-\Lambda^{n}$, for some integer $n$. It is obvious that as $\Lambda \rightarrow 1$, the set of points expressable as $1-\Lambda^{n}$ for some integer $n$ becomes dense in the segment $[0,1]$, i.e., any $0 \leq p<1$ can be approximated with an arbitrarily small difference by such a point, for $\Lambda$ sufficiently close to 1 . Therefore, $\lim _{\Lambda \rightarrow 1} Y_{\Lambda}(p)=p$ for any $0 \leq p<1$.

Now, denote the maximizing vector of $\Phi_{L}(s)$ by $\overrightarrow{p^{*}}=\left\langle p_{1}^{*}, \ldots, p_{M}^{*}, 0,0, \ldots\right\rangle$, and define the vector $\overrightarrow{p_{\mid N}} \triangleq\left\langle Y_{\Lambda_{N}}\left(p_{1}^{*}\right), \ldots, Y_{\Lambda_{N}}\left(p_{M}^{*}\right), 0,0, \ldots\right\rangle$, where $\Lambda_{N} \triangleq$ 


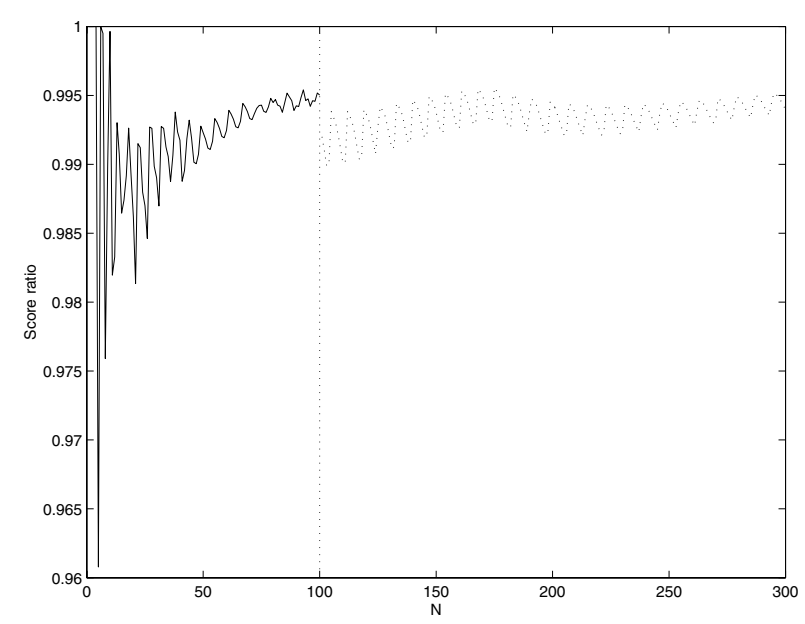

Fig. 4. Performance of the GI algorithm, relative to exhaustive search $(N \leq$ 100 , worst $L)$ and relative to continuous relaxation $(N>100, L=0.5)$.

$\left(L^{s}\right)^{1 / N}$. Define also the corresponding vector $\overrightarrow{n_{\mid N}} \triangleq$ $\left\langle\left\lfloor\log _{\Lambda_{N}}\left(1-p_{1}^{*}\right)\right\rfloor, \ldots,\left\lfloor\log _{\Lambda_{N}}\left(1-p_{M}^{*}\right)\right\rfloor, 0,0, \ldots\right\rangle$, and denote $N^{\prime}=\sum \overrightarrow{n_{\mid N}}$; note that $N^{\prime} \leq \sum_{i} \log _{\Lambda_{N}}\left(1-p_{i}^{*}\right)=$ $\log _{\Lambda_{N}} \prod_{i}\left(1-p_{i}^{*}\right)=\log _{\Lambda_{N}} L^{s}=N$.

Now, consider the score of $\overrightarrow{p_{\mid N}}$. It cannot be higher than $\mathrm{E}_{\Lambda_{N}}\left(N^{\prime}\right)$, since $n_{\mid N}$ is just one of the 'eligible' vectors over which $\mathrm{E}_{\Lambda_{N}}\left(N^{\prime}\right)$ is maximized. In light of Lemma 1 , it is therefore not higher than $\mathrm{E}_{\Lambda_{N}}(N)$ as well. Thus, $\mathrm{E}_{\Lambda_{N}}(N)$ is 'sandwiched' between the scores of $\overrightarrow{p_{\mid N}}$ and $\overrightarrow{p^{*}}$ (the latter, by definition, being simply $\Phi_{L}(s)$ ). However, since $\Lambda_{N} \rightarrow 1$ as $N \rightarrow \infty$, we have $\overrightarrow{p_{\mid N}} \underset{N \rightarrow \infty}{\longrightarrow} \overrightarrow{p^{*}}$, which finally implies $E_{\Lambda_{N}}(N) \underset{N \rightarrow \infty}{\longrightarrow} \Phi_{L}(s)$.

Unfortunately, we do not have a similar formal result for the approximation quality of algorithm GI; however, our experience shows that it generally achieves very good results. We have compared the score it attains with the one found by exhaustive search, for all $N \leq 100$ and all $L$ between 0.001 and 0.999 in increments of 0.001 . The solid line in Figure 4 shows the worst ratio between the scores, taken over all values of $L$, as a function of $N$. It can be seen that the score ratio does not drop below 0.96 (reached at $N=5, L=0.5$, where the score attained by GI is 1.53125 versus the optimal 1.59375), and it tends to get closer to 1 as $N$ increases. In fact, for $N \geq 43$, the score ratio never drops below 0.99 , for any $L$.

A significant related observation is that, for all $N$, the worst-ratio points always corresponded to $L$ around 0.5 (more precisely, $0.416 \leq L \leq 0.559$ ), while for 'extreme' values of $L$ (close to 0 or to 1 ), the GI algorithm converged much closer to the optimal score. ${ }^{\dagger}$ This can be intuitively explained by the fact that, for $L \approx 0.5$, the optimum is much less proclaimed, i.e., there exist many vectors with scores that are very close

\footnotetext{
${ }^{\dagger}$ In fact, it can be easily proved by induction that when the maximizing vector is either $\langle\underbrace{1, \ldots, 1}_{N}\rangle$ or $\langle N, 0, \ldots, 0\rangle$, algorithm GI converges to it correctly (we omit the formal proof here); note that the above are the limits of the optimal vector for $L \rightarrow 0$ and $L \rightarrow 1$, respectively.
}

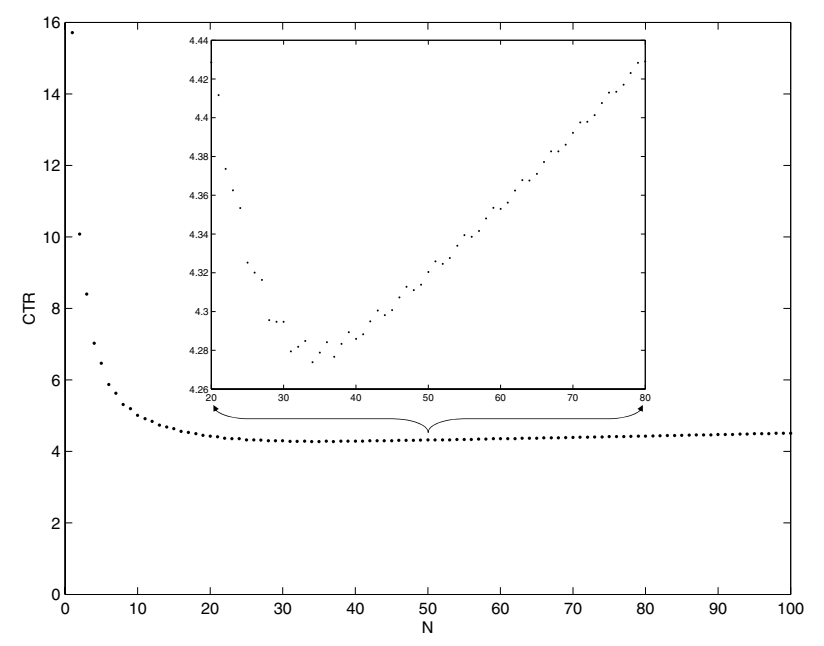

Fig. 5. The ratio $\frac{a \cdot T+b \cdot N}{\mathrm{E}_{L}(N)}$ as a function of $N$, for $a=10, T=1, b=1$, $L=0.3$. The inset 'zooms in' on $20 \leq N \leq 80$.

to the optimum.

Consequently, to evaluate the performance of GI for larger $N$ (for which exhaustive search is too time-consuming), it makes sense to plot the ratio between the score it attains for $L=0.5$ and the corresponding value computed by the continuous relaxation method; this estimates a lower bound on the score ratio between GI and the true optimum. This plot is shown as the dotted line in Figure 4 (and, incidentally, is similar for nearby values of $L$ as well). We conclude that algorithm GI, being arguably the cheapest imaginable in terms of computation complexity, attains a score at most $1 \%$ lower than the optimum, probably acceptable for most purposes.

\section{Finding the Optimal Window Size}

We now turn to discuss the solution of the 'outer problem', namely, finding the window size $(N)$ that minimizes the cost/throughput ratio (5). To begin, note that generic search algorithms (e.g. Fibonacci or golden-section search [16], [15]), with any of the methods outlined in the previous section as a 'subroutine' for computing $\mathrm{E}_{L}(N)$, are inefficient, as they neglect the internal redundancy between computations for different $N$. For example, observe that, in algorithm GI, $N$ only sets the total number of iterations, each of which by itself is independent of $N$. This raises the idea of proceeding with such iterations until the cost/throughput ratio (computed on the fly) ceases to decrease, instead of setting an advance limit.

Figure 5 shows a typical plot of the target ratio as a function of $N$. Observe that the function decreases steeply at first but quickly becomes quite 'flat', eventually rising slowly amid a somewhat noise-resembling behavior. ${ }^{\ddagger}$ This shape is indeed expected, considering that the ratio expression is $\Theta\left(\frac{a \cdot T+b \cdot N}{N / \log _{1 / L} N}\right)$ (recall Theorem 2): thus, for small $N$

\footnotetext{
$¥$ The plot in Figure 5 was obtained with the exact values of $\mathrm{E}_{L}(N)$, found by an exhaustive search; however, the plot shape is essentially similar if any of the approximate evaluation methods is used instead.
} 
Initialization: Set $\vec{n}=\langle 0,0, \ldots\rangle, N \leftarrow 0$, Best_CTR $\leftarrow$ L Loop:

$N \leftarrow N+1$

For every $j$ such that either $j=1$ or $n_{j-1}>n_{j}$ :

Temporarily set $n_{j} \leftarrow n_{j}+1$

Compute the score of $\vec{n}$

Restore $n_{j}$

For the $j$ that got the best score:

Set $n_{j} \leftarrow n_{j}+1$

Set $C T R \leftarrow$ the cost/throughput ratio for $\vec{n}$

If $C T R<B e s t \_C T R$

Set Best_CTR $\leftarrow C T R, N^{*} \leftarrow N$

If $N=2 N^{*}$, terminate; else go back to Loop.

Fig. 6. Algorithm Greedy-Outer (GO).

$\left(N \ll \frac{a T}{b}\right)$, it decreases at a rate of $\frac{1}{N / \log _{1 / L} N}$, while for $N \gg \frac{a T}{b}$, it increases at a rate of $\log _{1 / L} N$, i.e., much more slowly. The noise-like non-monotonocity, especially apparent around the minimum point, is due to combinatorial effects that we do not go into further; however, it may cause a potentially large number of 'false' local minima (e.g. $N=$ $\{29,31,34,37,40,44,48, \ldots\}$ in Figure 5), requiring care to avoid terminating the search prematurely.

To decide on an appropriate termination condition, we tested the algorithm for all $L$ between 0.001 and 0.999 in increments of 0.001 , with $b=1, T=1$, and $a \in$ $\{1,2, \ldots, 10,20, \ldots, 100,200, \ldots, 1000\}$ (recall that, for a given $L$, the optimal $N^{*}$ depends only on $\frac{a T}{b}$ ). We point out that this range covers all the practically interesting cases: for $\frac{a T}{b}<1$, the optimal window size rarely gets above 1 , while for $\frac{a T}{b}=1000$ the search already reaches window sizes of many thousands of packets. In all these runs, we found that, similarly to Figure 5, the local minima indexes formed nearly arithmetic sequences with periods much smaller than $N^{*}$ itself (in a few cases, there were two separate regions of local minima sequences with different periods, both much smaller than the corresponding $N^{*}$ ). A simple termination condition that is based on the above observation is $N=2 N^{*}$, i.e., stop the search after completing twice the iteration number in which the optimum was found. Figure 6 describes the algorithm with this condition employed; this algorithm, termed GO (for "Greedy Outer"), did not fail to find the global minimum even in a single instance. Admittedly, this condition is quite conservative; however, considering that the optimal strategy computation is performed off-line and infrequently, and that the best strategy found so far can be employed even before the search is completed, perfecting the termination condition to reduce the computation by a constant factor at most does not seem to be of major importance. ${ }^{\dagger}$

Finally, Figure 7 plots the optimal cost/throughput ratio as a function of $a$, for a few select values of the loss rate; note that the horizontal axis is logarithmic. These plots clearly

\footnotetext{
${ }^{\dagger}$ We point out that termination criteria based on the target value itself, rather than the window size (such as "stop when the current target value has risen to 5\% above the optimum so far"), also work, but may lead to exponential complexity, due to the logarithmic increase rate of the target expression for large $N$.
}

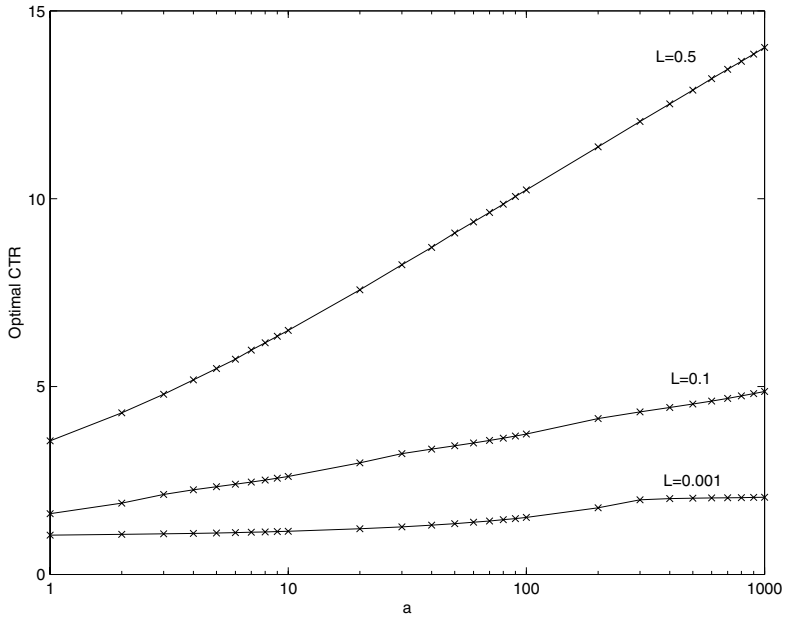

Fig. 7. Optimal cost/throughput versus $a$ for several loss rates.

demonstrate the property predicted by Theorem 4, namely, that the ratio increases logarithmically in $a$.

We close this section with a conclusive example that demonstrates the performance of the GO algorithm, as well as other techniques presented earlier.

Example: For the parameter values depicted in Figure 5 (namely, $L=0.3, T=1, b=1, a=10)$, algorithm GO finds the strategy $\langle 3$, $3,3,3,3,3,3,3,2,2,2,2,1,1,0,0, \ldots\rangle$, at $N^{*}=34$. It has a score of 10.295 , which leads to a cost/throughput ratio (i.e. average cost per successfully communicated packet) of 4.2739 . Incidentally, the greedy search happens to find the optimal strategy in this case; no further improvement can be gained by exhaustive search.

For comparison, the optimal window size with 'classic' sliding windows, found by formula (7), is 5 (i.e., the strategy is $\langle 1,1,1$, $1,1,0,0, \ldots\rangle$ in our terms), with a corresponding cost/throughput value of 7.7273 . Thus, using a strategy with advance retransmissions nearly halves the average cost per packet.

Let us now try $a=100$, with the other parameters as before. This time, GO finds $N^{*}=531$, with the strategy $\langle\underbrace{6, \ldots, 6}_{12}, \underbrace{5, \ldots, 5}_{66}$, $\underbrace{4, \ldots, 4}, \underbrace{3, \ldots, 3}, 2,2,2,2,1,1,0,0, \ldots\rangle$. Its score is 97.6449 , and 23 corresponding

cost/throughput value is 6.4622 . The 'classic' optimal window size here is 10 , yielding a cost/throughput value of 48.513 ; thus, in this case, the advantage of using a strategy with retransmissions is much greater. In fact, it can be seen that the cost/throughput increased only mildly from the previous case, despite the tenfold raise of the time cost, due to using a significantly larger window; this resulted in a nearly-tenfold increase in the throughput as well, which, therefore, nearly canceled the extra time cost.

It is interesting to note that, this time, the strategy found by GO is not optimal. While exhaustive search for the inner problem is unfeasible for $N=531$, a local search around GO's result found the strategy $\langle\underbrace{6, \ldots, 6}_{10}, \underbrace{5, \ldots, 5}_{68}, \underbrace{4, \ldots, 4}_{24}, \underbrace{3, \ldots, 3}_{9}, 2,2,2,1,1,0,0, \ldots\rangle$, with a slightly higher score of 97.651 . The continuous relaxation method in this case results in an upper bound of 98.6863 for the score; therefore, the strategy obtained by greedy search, with a score of 97.6449 , cannot be off by more than about $1 \%$ from the 'truly' optimal one. 


\section{CONCLUSION}

We have investigated optimal sliding-window strategies in network connections where the packet transmission time is negligible compared to the round-trip delay. We associated a cost per unit of time and per packet transmission with the connection, and defined the optimal strategy as one that minimizes the expected cost/throughput ratio. We derived several important bounds on the optimal strategy performance; specifically, for a window size of $N$, we showed the number of successful in-order packets to be $\Theta\left(\frac{N}{\log N}\right)$, and used this result to prove that the cost/throughput ratio increases logarithmically in the time price. We then studied practical solution algorithms, and found that strategies that are suboptimal by only a few percent can be computed with a very efficient 'greedy' algorithm. Our approach was demonstrated to attain a significantly lower cost/throughput ratio than 'classic' sliding windows, where a packet is retransmitted only after a timeout or negative acknowledgment.

Our attention was limited to strategies that use simple retransmissions only; however, as explained in the Introduction, the methodology can be extended for FEC coding as well. The optimization problem in that case is somewhat more complex (it involves an extra parameter, namely the size of the coding block), yet its solution follows essentially the same approach, except that the strategy score expression is based on the coding redundancy rather than number of retransmissions. In particular, the optimal strategy can be expected to use a higher-redundancy coding for the first packets in every window than for later ones.

Similarly, our technique can be extended to the case that the receiver has a buffer capable of accepting packets out of order, and reports its state in the acknowledgments (this is known as a receiver capable of selective repeat). Instead of a single vector specifying the number of retransmissions for each packet, the optimal strategy in this case is described by a set of such vectors, corresponding to the possible buffer states and specifying the optimal sequence of tranmissions for each state. Still, the computation of these vectors involves the optimization of essentially the same score expression. Furthermore, the strategy remains invariant to the next-expected packet index and thus can be considered to be of the slidingwindows type.

The strategies discussed in this paper were assumed to wait for all the acknowledgments from a window before setting out to transmit the next one. We explained, while presenting the general model, why such behavior is optimal if the packet transmission time is neglected. In reality, of course, a packet transmission takes a certain time $t_{x}>0$. This can be simply catered to by replacing the packet transmission price $b$ with $b+a \cdot t_{x}$, i.e. including the extra per-packet cost due to the time it takes to transmit it, with no further changes in the solution algorithm. Strategies thus computed are adequate if the connection's delay-bandwidth product is large (and, hence, $\left.t_{x} \ll T\right)$. Otherwise, i.e. if a packet transmission takes a significant fraction of the round-trip time, it may be better not to wait for all acknowledgments from the previous window, and proceed with transmission with only a partial information on previous successes and losses. Then, a strategy is no longer described by a vector applied at every multiple of the roundtrip time, but, rather, by a rule applied after every packet transmission and specifies the packet most worthwhile to transmit next (if at all), according to the information available up to that moment. The investigation of optimal strategies and their properties in this framework, as well as further development of the other extensions outlined above, form a subject for future work.

\section{REFERENCES}

[1] A. Tanenbaum, Computer Networks, 3rd ed. Upper Saddle River, NJ: Prentice-Hall, 1996.

[2] "ISO/IEC standard 13239:2000 (HDLC procedures)," Feb. 2002.

[3] M. Mathis, J. Mahdavi, S. Floyd, and A. Romanow, "RFC 2018: TCP selective acknowledgment options," Oct. 1996.

[4] S. Floyd and V. Jacobson, "Random early detection gateways for congestion avoidance," IEEE/ACM Transactions on Networking, vol. 1, no. 4, Aug. 1993.

[5] L. Libman and A. Orda, "Optimal timeout and retransmission strategies for accessing network resources," IEEE/ACM Transactions on Networking, vol. 10, no. 4, Aug. 2002.

[6] M. Allman, S. Dawkins, D. Glover, J. Griner, D. Tran, T. Henderson, J. Heidemann, J. Touch, H. Kruse, S. Ostermann, K. Scott, and J. Semke, "RFC 2760: Ongoing TCP research related to satellites," Feb. 2000.

[7] M. Allman, C. Hayes, H. Kruse, and S. Ostermann, "TCP performance over satellite links," in Proc. 5th International Conference on Telecommunication Systems, Nashville, TN, Mar. 1997.

[8] C. Barakat, N. Chaher, W. Dabbous, and E. Altman, "Improving TCP performance over geostationary satellite links," in Proc. IEEE Globecom, Dec. 1999.

[9] E. Altman, K. Avrachenkov, and C. Barakat, "TCP network calculus: The case of large delay-bandwidth product," in Proc. IEEE Infocom, New York, NY, June 2002.

[10] D. Katabi, M. Handley, and C. Rohrs, "Internet congestion control for future high bandwidth-delay product environments," in Proc. ACM SIGCOMM, Pittsburgh, PA, Aug. 2002.

[11] K. Park and W. Wang, "AFEC: An adaptive forward error correction protocol for end-to-end transport of real-time traffic," in Proc. 7th International Conference on Computer Communications and Networks $(I C C C N)$, Lafayette, LA, Oct. 1998.

[12] C. Barakat and E. Altman, "Bandwidth tradeoff between TCP and linklevel FEC," in Proc. IEEE International Conference on Networking, Colmar, France, July 2001.

[13] B. Liu, D. Goeckel, and D. Towsley, "TCP-cognizant adaptive forward error correction in wireless networks," in Proc. IEEE Infocom, New York, NY, June 2002.

[14] R. Corless, G. Gonnet, D. Hare, D. Jeffrey, and D. Knuth, "On the Lambert W function," Advances in Computational Mathematics, vol. 5, 1996.

[15] L. Libman and A. Orda, "Optimal sliding-window strategies in networks with long round-trip delays," CCIT Report \#384, Dept. of Electrical Engineering, Technion, Israel, May 2002. Available from http://www.ee.technion.ac.il/libman/papers/ccit384.ps.gz.

[16] D. Luenberger, Linear and Nonlinear Programming. Reading, MA: Addison-Wesley, 1984. 